\title{
Review of: "Ivermectin"
}

Matthew Elvey

Potential competing interests: The author(s) declared that no potential competing interests exist.

Certainly, ivermectin's antiviral and immunomodulatory effects, and perhaps its fibrinolytic and anti-cancer effects, warrant mentioning. 